\title{
Heart rate monitoring, activity recognition, and recommendation for e-coaching
}

\author{
Toon De Pessemier • Luc Martens
}

Received: date / Accepted: date

\begin{abstract}
Equipped with hardware, such as accelerometer and heart rate sensor, wearables enable measuring physical activities and heart rate. However, the accuracy of these heart rate measurements is still unclear and the coupling with activity recognition is often missing in health apps. This study evaluates heart rate monitoring with four different device types: a specialized sports device with chest strap, a fitness tracker, a smart watch, and a smartphone using photoplethysmography. In a state of rest, similar measurement results are obtained with the four devices. During physical activities, the fitness tracker, smart watch, and smartphone measure sudden variations in heart rate with a delay, due to movements of the wrist. Moreover, this study showed that physical activities, such as squats and dumbbell curl, can be recognized with fitness trackers. By combining heart rate monitoring and activity recognition, personal suggestions for physical activities are generated using a tag-based recommender and rule-based filter.
\end{abstract}

Keywords Heart rate · activity recognition · recommendation · e-coaching · health $\cdot$ mobile

\section{Introduction}

In modern society, obesity due to insufficient physical activity is an ever growing problem. Obesity can induce amongst others, diabetes, heart diseases and stroke, gallbladder disease, and gallstones. Research has shown that the majority of health care costs [5] are due to physical inactivity [6]. Recent research in

T. De Pessemier - L. Martens

Imec - WAVES - Ghent University

iGent - Technologiepark 15, 9052 Gent, Belgium

Tel.: +32-9-2643325

E-mail: toon.depessemier@ugent.be

E-mail: luc1.martens@ugent.be 
health care supports the theory that a healthy diet and regular physical activity are much more effective than traditional medication to cure diabetes [19]. Nutrition and training schedules are online available, but are often not personalized to the user's needs or physical capacities and are static without taking into account user's progress.

Thus, new efforts are made to decrease national obesity levels [25], thereby using technology such as multi-modal sensors, web frameworks, and data mining. Multi-modal sensors enable real-time monitoring of physical activities performed by the user. In the domain of public health monitoring, most of these sensor applications keep track of energy expenditure while performing daily activity $[9,12]$.

Since the Web 2.0 evolution, more formal and informal health information has become available, with the perspective of a new generation of wellinformed, healthy individuals. This phenomenon is often referred to as eHealth 2.0. eHealth 2.0 turns users into health information producers and consumers by offering a multitude of health information data $[15,10]$.

To cope with the problem of information overload incurred by Web 2.0 and its eHealth 2.0 counterpart, recommender systems are used as an effective information filter and at the same time as a tool for providing personal advice through suggestions $[41,8]$. The gathered health data can be used as input for such a lifestyle recommender that encourages users to adopt a more healthy way of life. Suggestions for a specific fitness activity or a running trail out of the many available alternatives, have to be tailored to the physical capabilities of each individual.

To assess the intensity and load of physical activities for a user, measuring merely the physical movement is insufficient, since the user's physical capabilities cannot be ignored. The combination of heart rate measurements and motion sensors allows to assess the user's physical limits and how intense the activities are experienced [17].

Most people do not have a specialized device for measuring heart rate at their disposal, but recent wearable devices are also capable of this. Popular mobile devices and wearables are equipped with sensors that promise to measure heart rate. However, the accuracy of heart rate measurements using these devices is still unclear. Manufacturers choose not to assert claims regarding the accuracy of the detection of (abnormal) beating patterns; otherwise their gadget would get classified as a medical device and would have to undergo FDA (Food and Drug Administration) regulatory scrutiny [26]. Therefore, the first research question of this paper is: how accurate are heart rate measurements with these devices in rest condition and during physical activities? The results of this study are important for (mobile) applications and services that rely on heart rate data from these devices.

Moreover, wearables offer some basic activity recognition, such as counting steps or calculating the speed of a running session, but the recognition of specific physical exercises is still missing. This study investigates automatic activity recognition. The second research question of this paper is: can activ- 


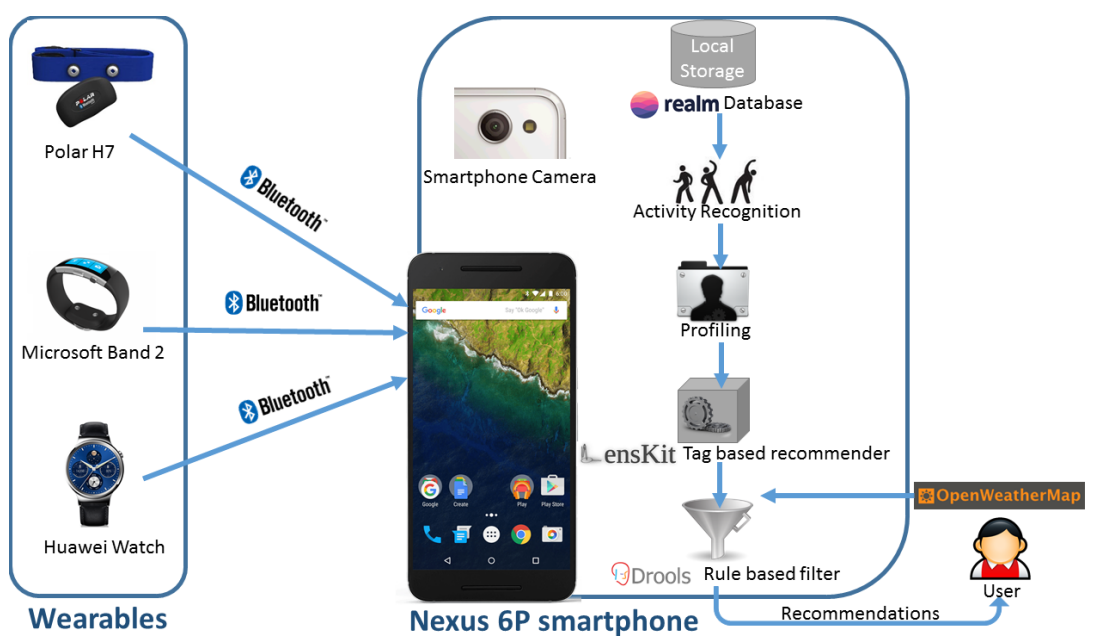

Figure 1 Diagram showing the data flow through the different components of the system

ity recognition help in e-coaching activities, e.g. by counting the number of repetitions of a performed exercise?

To answer these questions, an Android application has been developed to gather data of the accelerometer for activity recognition and monitor the heart rate of test subjects, simultaneously with different devices during various physical activities. Both, heart rate and activity recognition, are used as health information sources for a recommender system to suggest activities tailored to the user's preferences and capabilities.

Figure 1 shows the data flow through the different components of the system. Various wearables are connected to the Android mobile phone (Nexus $6 \mathrm{P})$ through a Bluetooth connection. Data of accelerometers and heart rate sensors are stored in the database. The data of accelerometers are processed and repetitions of specific movements are detected by activity recognition. The types of activities, the number of repetitions, and the intensity (speed of execution) are used for profiling the user. Based on this sports profile, a tag based recommender selects activities that match the user's preferences. Subsequently, a filtering with a rule based system is performed to match the intensity of the recommended activity to the physical capabilities of the user (as estimated by the measured heart rate). Also weather conditions are taken into account with these rules. The resulting activities are offered to the user as personal suggestions.

The remainder of this paper is structured as follows. Section 2 gives an overview of related work in the domain of eHealth and mobile health apps. In Section 3, existing methods for heart rate measuring are discussed. The various types of mobile devices for heart rate measuring are listed in Section 4. In Section 5, details about our measuring method and our experiences with the various devices are discussed. Section 6 provides the results of the measurement experiments. Section 7 discusses activity recognition for e-coaching 
purposes. Section 8 proposes a recommender system providing personalized suggestions for physical activities tailored to the user's preferences and physical capabilities. Finally, conclusions are drawn and future work is mentioned in Section 9.

\section{Related Work}

The domain of eHealth has been evolved thanks to two major influences: on the one hand the increasing availability of sensors for tracking physical activities, not only in smartphones but also in other devices, such as smart wearables; on the other hand the easy accessibility of health information, stimulating the users' interest for monitoring their physical condition.

Various health information systems (HIS) have been proposed to process heart rate data or recognize physical activities. These HIS can have three primary goals: inform, assist in the decision making, or convince the end-user. For instance in the HOMEY project, technology for innovative tele-medicine services is developed [3]. The goal of these services is to effectively manage an incremental dialogue between a patient and a tele-medicine system, taking into account user preferences, needs, and the time course of the patient's disease. More specifically, the HOMEY project focuses on automated, telephone-based home monitoring services for chronic hypertensive patients [18]. Patients are regularly asked to specify their heart rate and blood pressure values, and subsequently, suggestions for physical activities are provided, e.g., "Are you still swimming two times a week?" or health advice is offered, such as "You should stop smoking". This personalized dialog with the patient is based on goals and rules specified by medical staff. A clinical trial involving 300 hypertensive patients showed a blood pressure decrease in the group of patients using the HOMEY service. The study emphasizes the importance of the usability of the HIS to stimulate an intensive use. However, the HOMEY service is not able to automatically measure heart rate or detect physical activities, in contrast to our Android application.

As overaging is becoming a main societal challenge, many research initiatives are in the domain of Ambient Assisted Living (AAL). AAL systems help elderly to overcome typical difficulties of everyday life, and contribute positively to their well-being [20]. These systems often use sensors, capturing biosignals, e.g., a sleep monitoring mattress that analyzes breathing, heart rate, snoring, and body movement [2]. Measures of walking speed and amount of activity in the home can be obtained by accelerometers [22].

Since falls and the resulting hip fractures in the elderly are a major health problem, various studies regarding fall detection have been conducted. An effective fall detection system is required to provide urgent support [34]. Sensors are installed in the furniture or in a cane [46]. Moreover, preimpact fall detection allows the development of an inflatable hip pad for preventing fall-related hip fractures. A single sensor with the appropriate kinematics measurements 
and detection algorithms, located near the body center of gravity, is able to distinguish an in-progress and unrecoverable fall from non-falling activities [45].

An important characteristic of a health-related service is the intrusiveness. A camera can be used to detect age dementia [33], but is often considered as invasive by users. Our application tries to gain the user's trust by using only wearables and a smartphone, and being transparent about how and which data are used. If health-related data are used for other purposes or get into the wrong hands, the privacy of the users can be compromised. A data collector may disclose personal health information to untrusted parties. This could occur either on purpose, i.e., by selling the personal information to a third party (e.g., a health insurance company), or involuntarily through a security breach.

On the mobile platform, tens of thousands health apps are available [7], often called mHealth (Mobile Health) apps [21]. For that reason, the offer of health apps is even considered as an overload for medical professionals and consumers [42]. Both continue to express their concerns about the quality of many apps [7]. Besides, the importance of personalization strengthens the need to automatically acquire personal data, such as performed physical activities or heart rate.

However, tracking physical activities or measuring heart rate is complex and the accuracy is often unclear for general-purpose wearables. For commercially available breast belt measuring devices, evaluations in terms of accuracy have been performed [44]. However for recent wearable devices, only a limited number of studies investigated the accuracy of heart rate monitoring, often in specific conditions. In non-moving conditions, heart rate monitoring using a wrist-worn personal fitness tracker has been evaluated with patients in the intensive care unit [27]. The measured values were slightly lower than those derived from continuous electrocardiographic monitoring. The authors argued that further evaluation is required to investigate if personal fitness trackers can be used in hospitals, e.g. as early warning systems. Another study has investigated the accuracy of step counts and heart rate monitoring with wearables [14]. Test subjects were asked to walk a specific number of steps during the measurements. The accuracy of the heart rate measurements with the tested wearable devices showed to be very high. Our paper goes further by studying the accuracy of heart rate measurements during intense physical activities, and with various types of wearable devices.

To recognize different physical activities, various approaches based on video analysis have been published [31]. More recently, wearable sensors became popular for activity recognition. Classifiers based on hidden Markov models have been proposed for such wearable sensors [28]. Activities such as driving a car, riding a bicycle, walking, or standing still can be recognized. In recent versions of Android, similar functionality to recognize activities is available through Google's activity recognition API.

To classify activities such as walking, race walking, and running based on unlabeled data, an unsupervised method for recognizing physical activities using smartphone accelerometers has been proposed [32]. Two additional smartphones are attached to the upper arms of the user to recognize specific 
actions while playing basketball, such as passing or bouncing the ball, or a free throw. Although the hardware of the accelerometer might be very similar for wearables, the specific position of a wearable around the wrist can provide very different data reflecting movements of the hand and wrist on top of the arm movements.

Other studies investigated how simple actions can be used to recognize more complex activities, which are semantically more representative of a human's real life [29]. The algorithm is based on temporal patterns (such as actions occurring after other actions, or actions that overlap) and a multi-task learning approach [30].

In contrast, our research targets activities that cannot be classified based on the movement speed or temporal patterns, but involve typical body or arm movements, such as squats or dumbbell curl exercises. Our focus is on recognizing the number of repetitions in view of tracking the physical load, rather than on classifying the activities. Based on the physical load and personal preferences, personalized recommendations for activities are offered to the user.

The growing availability of health data has brought the problem of information overload [16] to the ehealth domain. For instance, too many sports schedules and diet plans are available, but only a minority are tailored to the specific needs of a person. This emphasizes the need to personalize health information and services, which is ongoing since the mid-90s [4] and is demonstrated in Computer-Tailoring Health Education Systems [16]. Personalization in the health domain is described as "adapting the content of the materials, with the aid of computers, to the specific characteristics of a particular person" [43].

Personalization can be achieved by using a recommender system. Personalized recommendations, tailored messages, and customized information have shown to be far more effective than the non-personalized alternative $[40,8]$. Health promotion and wellness driven applications often use collaborative filtering techniques to cope with the overload of health data and identify the most relevant information [23]. Collaborative filtering makes the selection of information based on actions of the community, and does not rely on a central agency or individual expert. As a result, the quality of the selection is depending on the size and engagement of the community using the service. Alternative content-based solutions do not depend on community activity, but require specific metadata to assess the suitability of information items. Our proposed recommender is a combination of a rule-based and content-based system to filter the content for the users based on their preferences and physical capabilities.

\section{Heart Rate Monitoring}

Various methods exist for heart rate monitoring. Two methods that are important for this study are electrocardiography and photoplethysmography. 
Electrocardiography (ECG) is the process of recording the electrical activity of the heart using electrodes placed on the skin [24]. These electrodes detect the tiny electrical changes on the skin that arise from the heart muscle's electrophysiologic pattern of depolarizing during each heartbeat. For medical applications, e.g. in hospitals, this technique is applied with 10 electrodes, placed on the patient's limbs and on the surface of the chest.

Photoplethysmography (PPG), also known as optical heart rate sensing, monitors heart rate based on the combination of photo diodes and LEDs [39]. Green light is absorbed by blood, hence its red color. When part of the body (e.g. a finger) is placed on top of a light source, the light is partially absorbed by the blood and partially reflected. The reflected light is captured by a photo diode. During a pulse of the heart, more light is absorbed and the photo diode detects a reduction in green light intensity. During periods between two pulses, a high intensity of reflected light is measured by the photo diode. Although a green LED provides the most accurate results, an infrared LED is often used since this consumes less energy. PPG is a cheap method for heart rate measurements but comes with some disadvantages. Motion artifacts have been shown to be a limiting factor for accurate results during exercises and free living conditions. Person-dependent variations may also cause distortions in the measurements. For instance, a different blood perfusion induces a different absorption of light, which can result in deviating measurements.

\section{Mobile Devices}

For measuring heart rate, four types of devices can be distinguished. A device of each type was used in this study.

\subsection{Specialized Device}

Measuring hart rate is the main purpose of this type of devices, which often have only a limited number of sensors and a limited number of features. Typical specialized devices are pulse-oximeters, hart rate chest straps, and blood pressure monitors. These devices are often approved for medical use. Therefore, this device type is used as a reference in this study. As a specialized sports device, we used the Polar H7 in this study. This is a popular heart rate chest strap, which produces very accurate measurements (correlation of 0.97 with true heart rate [1]). To verify the accuracy in a home environment, we compared the heart rate measurements of the Polar $\mathrm{H} 7$ to the measurements of a specialized device that is approved for medical purposes, the Omrom M6 Comfort [35]. The Omrom M6 is a blood pressure monitor, which has to be attached around the upper arm for measuring the heart rate. A test with two users, at two different times, resulted in heart rate measurements of the Polar device with a precision similar to the Omrom (Table 1 ). Since a blood pressure monitor is rather expensive and less practical during sports activities, 
the Polar $\mathrm{H} 7$ was used as a specialized device during physical activities and considered as the reference device.

\subsection{Fitness Tracker}

These devices, typically worn around the wrist, measure movements and behavior, such as the number of steps taken, sleeping patterns, and sports activities, e.g. a light jog or a mad sprint. Fitness trackers are equipped with multiple sensors, such as a 3 -axis accelerometer to track movement in every direction, an altimeter to measure altitude and track the travelled height, and sometimes a gyroscope to measure orientation and rotation. These devices are typically cheaper than the specialized devices, but seldom approved for medical purposes. In this study, the Microsoft Band 2 was chosen as fitness tracker because of two reasons. It allows real time analysis of sensor data and Microsoft provides a comprehensive API. The API offers functionality such as aggregating the results of a query, thereby shifting the computational load to the Microsoft servers.

\subsection{Smart Watch}

As is the case with fitness trackers, smart watches are equipped with various sensors but are not medically approved. Whereas the main focus of fitness trackers is tracking physical activities, the goal of smart watches is more general and comprises tracking physical activities, informing users, and serving as fashionable device as extension of the smartphone. From a commercial viewpoint, the target group of customers is not limited to sports people, but includes also a broader group of people who like the design, or the extra features of a smart watch. Smart watches often have more hardware capabilities than fitness trackers (e.g., more processing power, color screen), allowing to extend their functionality with additional apps. In this study, the Huawei Watch was used as smart watch for the measurements because of its popularity and typical smart watch characteristics (e.g. Android Wear). To capture heart rate data in real time, a special Android Wear app was developed for the Huawei Watch. This app communicates with our Android app running on the smartphone through the Wearable Data Layer API.

\subsection{Smartphone}

By using (hardware-specific) apps, smartphones are able to measure heart rate using the built-in camera and LED flash based on PPG techniques. So, the hardware components of the device get a secondary feature, i.e. measuring heart rate, besides their main purpose, i.e. taking photos. In this study, we used the Google Nexus 6P smartphone and the camera and LED of this device were used to measure the heart rate. 


\section{Measurement Method}

\subsection{Experimental Setting}

To gather and store heart rate measurements of the four device types, an Android app was developed and deployed on a Google Nexus 6P smartphone. Figure 2 shows a screenshot of this app. The wearable devices have a Bluetooth communication link with this smartphone and the app has a separate service running for each device to transfer the raw data to the smartphone and store the data in a Realm IO database. Realm is an alternative solution for SQLite and delivers real time performance [38]. The evaluations of the heart rate measurements have been performed in a controlled environment.

Two test users of 22 years old were asked to use the wearables and perform some specific activities. The first test subject (male) has a low natural heart rate, whereas the second test subject (female) has a rather high heart rate in rest condition. Demographic and physical characteristics have an influence on the absolute value of the heart rate, but this research focuses on the changes and variations in the heart rate, rather than on the absolute value of the heart rate. Before each experiment, a rest period of 10 minutes was imposed to avoid influence of previous activities and the coupled heart rate.

The measurements of the heart rate during rest condition (Table 1) were performed in a home, indoor environment. Test users were asked to sit in a chair, doing nothing, while the devices measure their heart rate.

For evaluation of the heart rate measurements during physical activities, four exercises are performed in a fitness room by the two test subjects. Since similar results are obtained for these two test subjects, Figure 3 shows only the measurements of test subject 1 . The stair master is an exercise typically performed at the gym that simulated stair climbing on a machine. The leg press is a compound powerlift that engages most of the muscle groups in the legs. The dumbbell curl is an exercise that focuses on bicep workout. Longwalking simulates a walking activity in the fitness room. Because of practical reasons, measuring heart rate with the Omrom M6 was not possible during physical activities in the fitness room.

For heart rate measurements during physical activities, we witnessed a totally different user experience with the four devices.

\subsection{Specialized Device Experience}

The Polar device with chest strap is comfortable during sports, while holding its position on the chest. No difficulties or uncomfortable positions of the device were experienced during the test. The main goal of the device is measuring heart rate during sports, which is reflected in an excellent user experience. 


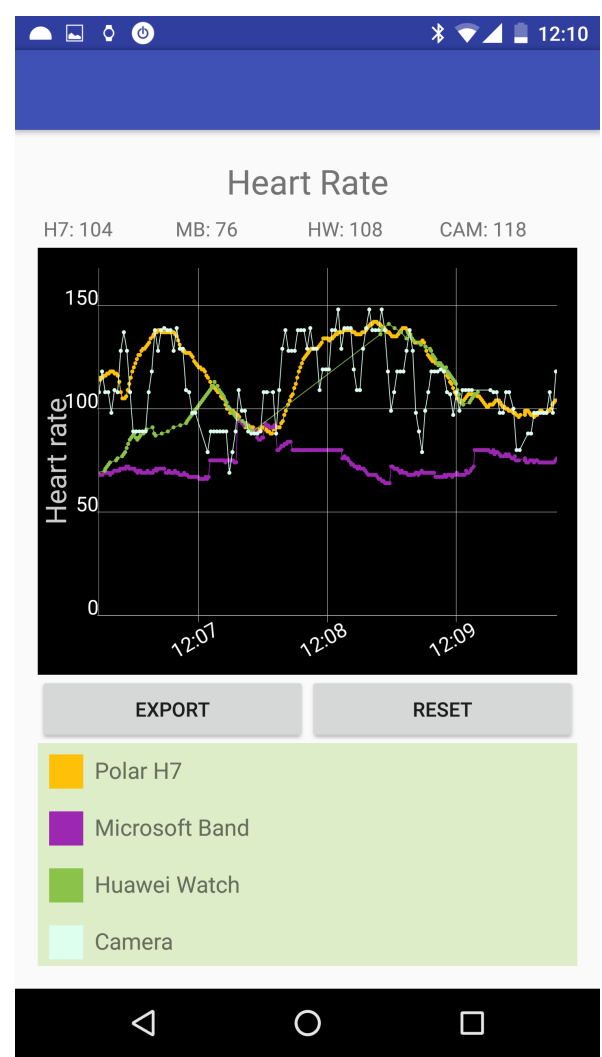

Figure 2 Screenshot of the mobile app for gathering heart rate data

\subsection{Fitness Tracker Experience}

Although the Microsoft Band 2 is a fitness tracker designed for sports activities, we do not experience it as ideal for heart rate measurements from a practical viewpoint. The strap of the device hinders when the wrist moves during activities.

\subsection{Smart Watch Experience}

During the usage of the Huawei Watch, we witnessed that motions of the wrist disturb the measurements, even if the strap is tightened excessively. The sensor in the smart watch loses the reference point, heart rate measurements are interrupted and no new data samples are obtained. To cope with this problem, our developed Android Wear app starts to recalibrate as soon as the measuring process is interrupted. Also variations in light intensity of the environment showed to disturb the measurement process. If the wrist is moved 
Table 1 Mean $\bar{x}$, standard deviation $\sigma$, and median $\tilde{x}$ of the heart rate measurements in rest condition with two users at two different times

\begin{tabular}{l|l|l|l|l|l|l|l|l|}
\multirow{2}{*}{ Device } & \multicolumn{2}{|c|}{ User 1 - Test 1 } & \multicolumn{2}{|c|}{ User 1 - Test 2} & \multicolumn{2}{l|}{ User 2 - Test 1} & \multicolumn{2}{c|}{ User 2 - Test 2 } \\
\cline { 2 - 8 } & $\bar{x} \pm \sigma$ & $\tilde{x}$ & $\bar{x} \pm \sigma$ & $\tilde{x}$ & $\bar{x} \pm \sigma$ & $\tilde{x}$ & $\bar{x}$ & $\tilde{x}$ \\
\hline Omrom M6 & $55 \pm 2.8$ & 55 & $58 \pm 2.9$ & 58 & $76 \pm 2.5$ & 76 & $84 \pm 4.2$ & 84 \\
Polar H7 & $56 \pm 1.7$ & 56 & $59 \pm 1.4$ & 59 & $77 \pm 3.0$ & 76 & $80 \pm 3.7$ & 79 \\
Microsoft Band & $50 \pm 2.9$ & 50 & $64 \pm 6.0$ & 64 & $75 \pm 3.3$ & 75 & $76 \pm 1.7$ & 76 \\
Huawei Watch & $55 \pm 2.0$ & 55 & $55 \pm 2.0$ & 56 & $73 \pm 3.3$ & 73 & $72 \pm 3.2$ & 71 \\
\hline
\end{tabular}

to a position where more or less light can be absorbed, measurements turned out to be invalid.

\subsection{Smartphone Experience}

Measuring heart rate using PPG on a smartphone induces four practical difficulties. Firstly, the PPG technique is influenced by personal characteristics of the user, such as properties of the blood and the size of the finger. Secondly, PPG is very sensitive to motion. Ideally the user's finger should be on top of the flash LED and photo diode in a static position without any movement at all. This requirement complicates heart rate measurements during physical activities. Thirdly, the prolonged use of the flash LED and camera heats up the phone excessively. The device gets so hot that it is impossible to cover the LED and camera with a finger for a period longer than a few seconds. Fourthly, the usage of LED and camera for heart rate measurements causes battery drainage. We witnessed a $4 \%$ decrease of the battery level during each measurement of 5 minutes in our experiments.

\section{Measurement Results}

Table 1 shows the resulting heart rate measurements for the two test subjects in rest condition. The smartphone with PPG technique is not included, since this method is highly influenced by personal characteristics of the test user. The measurements are repeated at two different times for the two test subjects. For each device, the mean, standard deviation, and median are listed in Table 1, which shows that all devices provide consistent results. The mean values and small standard deviation show that in rest condition, heart rate measurements obtained with these devices can be considered as reliable. The measurements of the Omrom M6, which is medically approved, are considered as the correct heart rate. The measurements of the Polar $\mathrm{H} 7$ are the most similar to the measurements of the Omrom M6.

Figure 3 shows the heart rate measurements obtained with the different devices for four different physical activities, respectively stair master, leg press, dumbbell curl and longwalking. For each device, trend and reactivity are investigated. Trend evaluates a scoped-out-view of the heart rate signal based on the statistics average and correlation. Reactivity is stricter and judges the 


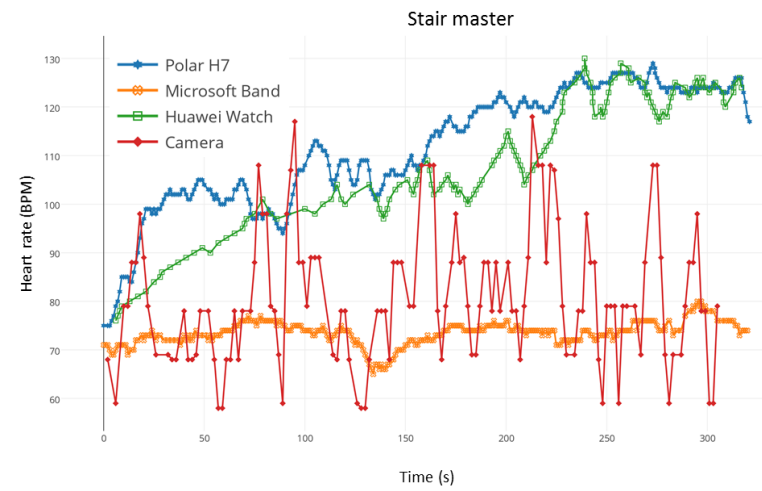

(a)

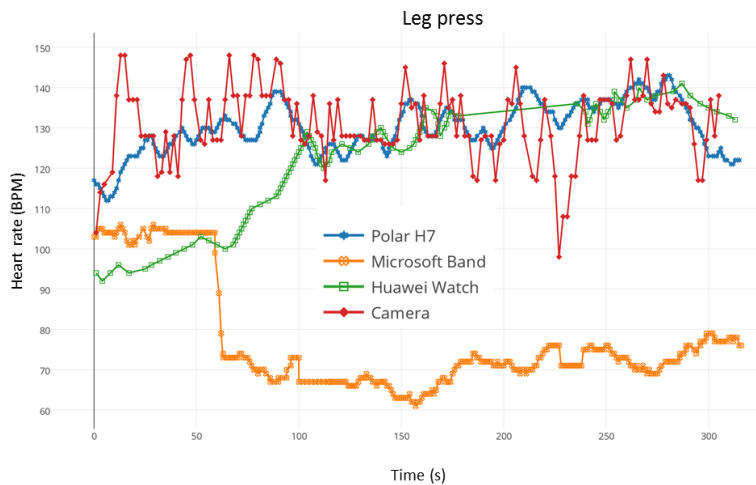

(b)

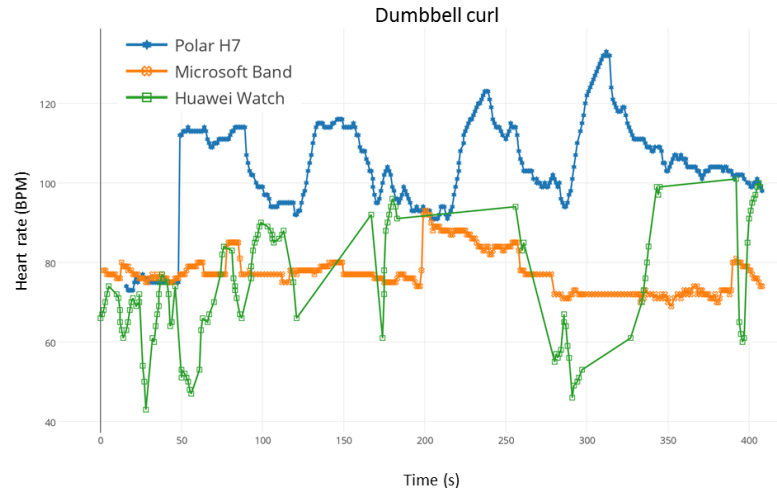

(c)

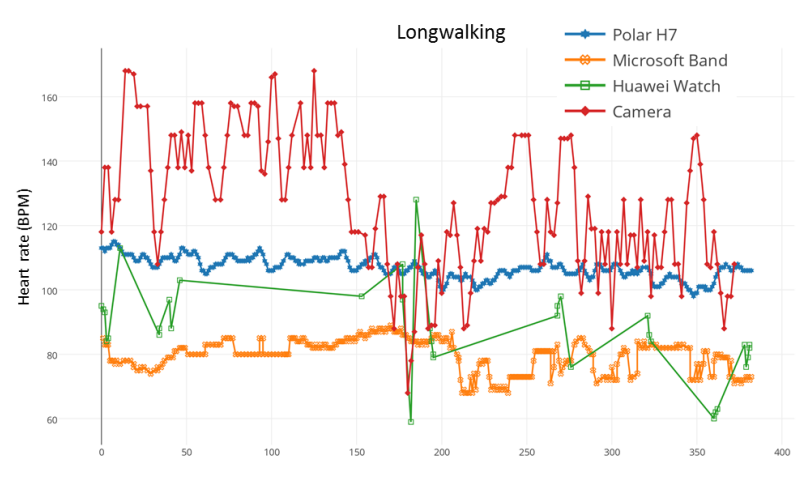

(d)

Figure 3 Heart rate measurements during physical activities. 
measurements during short periods of time. If an intense physical activity causes a sudden increase in heart rate, registering this increase without delay is important. If a heart rate sensor is able to detect these sudden changes quickly, then it can be considered as highly reactive.

\subsection{Specialized Device Measurements}

For the measurements during physical activities, the Polar H7 is considered as a reference device. The output of Polar is compared to the heart rate registered with the other devices.

\subsection{Fitness Tracker Measurements}

Regarding the trend, Figure 3 shows that the heart rate registered by the Microsoft Band 2 is consistently lower than the reference value of the Polar. This discrepancy varies for different heart rate values, which makes it hard to correct. Regarding the reactivity, measurements of the Microsoft Band deviate substantially from the reference during periods of intense activities. Figure 3(c) illustrates the low reactivity of the sensor: rapidly varying heart rates are not detected during the dumbbell curl exercise.

\subsection{Smart Watch Measurements}

Compared to the Polar H7, less measurement samples are obtained with the Huawei Watch, as visible in Figure 3(c) and 3(d). Movements of the device around the wrist cause interruptions in the measurement process, which might be a problem for detailed heart rate monitoring. Time periods without measurements correspond to periods of sensor (re)calibration due to these movements. As a result, this device shows to be less suitable for measuring heart rate during activities that involve a lot of movement of the wrist. Figure 3(a) shows a trend that corresponds to the reference of the Polar. The mean value is 5 beats per minute less than the mean value of the Polar reference. This difference is consistent for different heart rates. This allows a correction by adding the fixed difference to the measurements. Regarding the reactivity, Figure 3(c) shows the smart watch has some difficulties in detecting heart rates with varying intensities. Intensive periods are noticeable in the measurement data. But comparing the Polar and Huawei reveals a delay in the peaks of the data.

\subsection{Smartphone Measurements}

Measuring heart rate with the smartphone is not possible during dumbbell curl exercises because of the physical movements. Fluctuations in the measurements can be witnessed, even for a stable heart rate, because of the hardware dependencies, the environment, and the method itself. The trend of the 
PPG method may significantly differ from the Polar reference, as visible in Figure 3(a) and 3(d). For the leg press exercise (Figure 3(b)), the trend of the PPG method matches with the measurements of the Polar device. Regarding the reactivity, sudden increases in the heart rate are difficult to distinguish from the measurement fluctuations.

\section{Human Activity Recognition}

Many studies tackle the challenge of activity recognition by using multiple sensors placed on different parts of the body, e.g. on the chest and on the hip, composing a body sensor network [37]. However, the placement of these sensors can be considered too expensive and intrusive for daily (sports) activities. Therefore this work focusses on activity recognition using wearables. The goal is assisting the user in coaching tasks such as counting the number of performances of an exercise, rather than classifying the physical activities by type. This detection is based on real-time processing of the raw data of the accelerometer of the device. As a result, we focus on activities that involve movement of the arm or wrist. Activities with the arm and wrist in a rather static posture are not possible to recognize using accelerometer data. E.g., during the stair master activity, users typically hold the handles thereby producing no significant acceleration data. For each specific activity with movement of the arm or wrist, the primary axes of motion are determined and peaks in the data are detected. Because of specific characteristics of different physical activities, a custom approach was adopted for each activity.

\subsection{Squats}

The squat is a compound, full body exercise that trains primarily the muscles of the thighs, hips, buttocks, and hamstrings. Recognizing squats is based on the measured energy expenditure and a dominant motion along the Z-axis in the earth's coordinate system (due to vertical movement of the body). The energy expenditure, E, is calculated as the square root of the sum of the squared values of the acceleration along the three axes, x, y, and z. These values are calculated on different moments in time, $i$, and subsequently, the average is calculated.

$$
E=\frac{1}{N} \sum_{i=1}^{N} \sqrt{x_{i}^{2}+y_{i}^{2}+z_{i}^{2}}
$$

Individual performances of the squat activity are recognized if energy expenditure exceeds a threshold value while the condition of the dominant motion along the Z-axis is valid. To avoid a false double detection of the activity, an additional time frame is used. Since executing a squat properly requires some time, only one recognition of the activity is possible during the time 


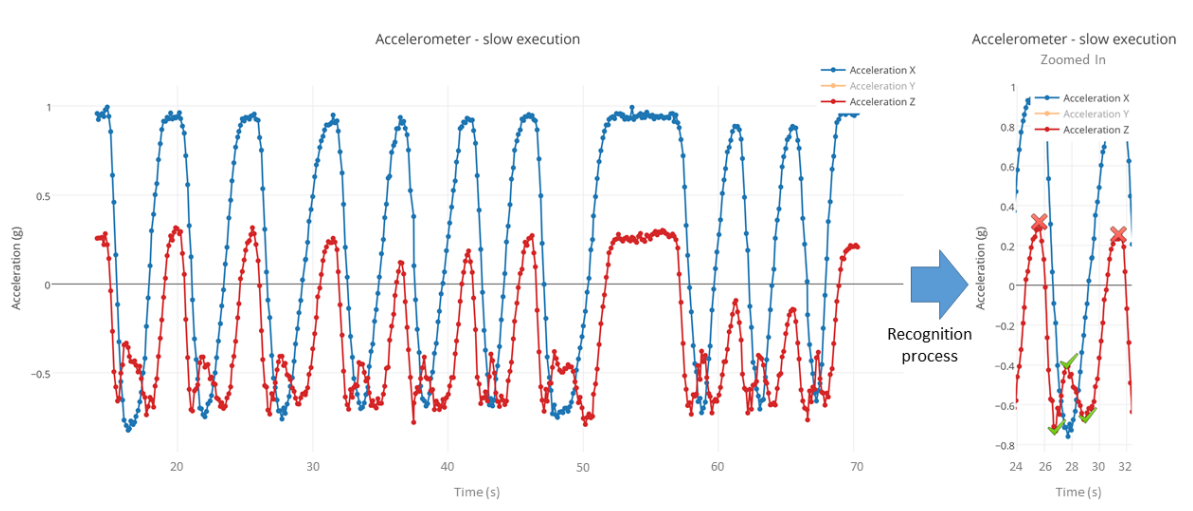

Figure 4 Data of the accelerometer during a dumbbell curl exercise with local optima

frame. The value of the time frame and the threshold are empirically determined based on samples of the activity.

\subsection{Dumbbell Curl}

The dumbbell curl is a typical activity to train the biceps. This activity is recognized by the specific pattern in the data of the accelerometer. Figure 4 shows the pattern of the accelerometer data that is typical for this exercise. Although the absolute values generated by the accelerometer can have a large variability depending on the execution speed of the activity and the body characteristics of the user, the pattern of local minima and maxima can be witnessed for every repetition. Therefore, the recognition process is based on the detection of sequences of local minima and maxima on the $\mathrm{X}$ and Z-axis. For each repetition of the dumbbell curl activity, 5 local optima on the Z-axis and 3 on the $\mathrm{X}$-axis can be witnessed. The red crosses denote the beginning and end of the activity, the green check marks indicate the intermedia optima. The detection of these local optima makes this recognition method directly usable for different variations of the dumbbell curl such as concentration curl, hammer curl, and barbell curl. In contrast, alternative recognition methods based on machine learning techniques require a large amount of training samples to recognize all these variations.

\section{Recommendation}

Suggestions for new activities, tailored to the user's preferences, physical capabilities and current progression, are an important aspect of e-coaching. Figure 5 shows a screenshot of the recommended activities for one of the users. Based on previously performed activities and measured heart rates, new activities are recommended to the user using a pipeline design (Figure 6 ), consisting of two subsequent components, running on the smartphone. 


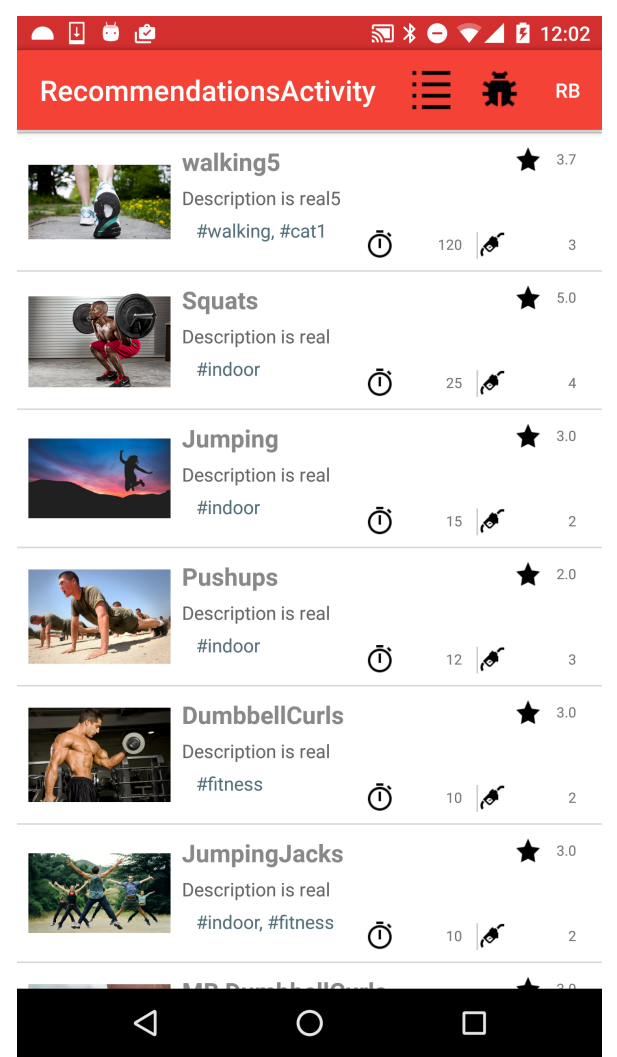

Figure 5 Screenshot of the application showing the recommended activities

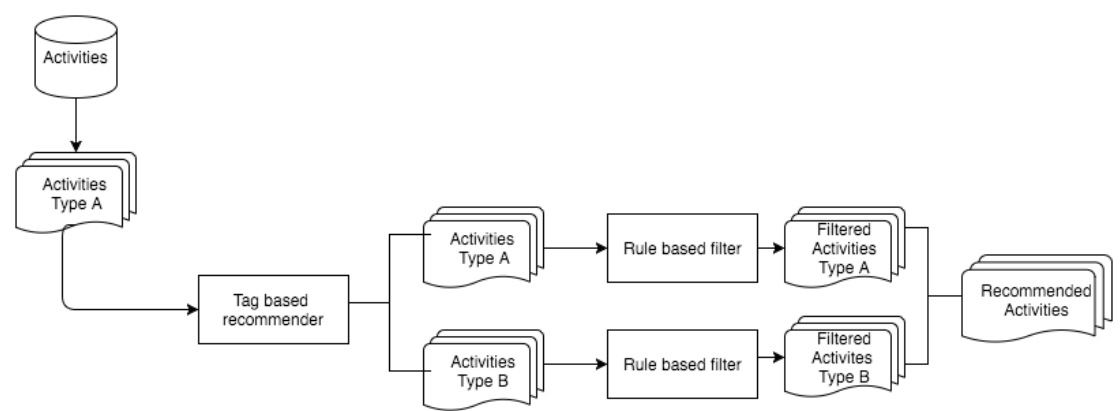

Figure 6 Schematic overview of the recommendation process consisting of a tag based recommender and a rule based filter

\subsection{Tag Based Recommender}

First, a content-based recommender selects activities that the user likes based on matching the tags of new activities and previously performed activities. This recommender, based on the LensKit [13] recommender toolkit, ensures 
that recommended activities are in line with the user's preferences. Preferences are derived from the user's ratings of activities (explicit feedback) and the selection and performance of a physical activity (implicit feedback). Rating an activity is performed by swiping of the recommended activities in the user interface (swiping to the left for a negative rating; to the right for positive feedback).

\subsection{Rule Based Filter}

Second, activities of each type are processed by a specialized rule based filter. This rule based filter selects the most suitable activities based on characteristics typical for that type of activities, .e.g. the distance for a running activity. A separate rule based filter is used for each type of activity to take into account the user's experience level. E.g., a user might be very good at running, and receive recommendations for intense running activities (pro-level). Meanwhile, the same user can be a beginner in the gym. So, the user's body is not trained for intense dumbell curl activities and recommendations at the beginner level will be appropriate here. For the rule based functionality, the system uses Drools [11], i.e. a business rules management system with business rules engine that is scalable and extendible through the use of drl files. Various rules are used to make the resulting recommended activities more suitable. E.g., no outdoor running activities are recommended in case of rainy weather. The OpenWeatherMap.org REST API [36] is used to retrieve weather data at the user's location.

The tag-based recommender eliminates many irrelevant activities in the first phase. As a result, the computational load of the rule-based filters is limited compared to the case of the reversed order (first rule based filter and then tag based recommender). This is important for the performance of the complete recommender system that is running on the smartphone.

\subsection{Relating Heart Rate and Activity}

During the performance of the physical activities, the heart rate is monitored. The goal is to coach the user thereby maintaining a healthy heart rate. Each physical activity has a range of the heart rate that can be expected during the performance. If the measured heart rate is out of this range, it is indicated on the screen of the wearable in red. After performing an activity, the recommender evaluates the performance as too intense, to easy, or just good. This evaluation, based on the measured heart rate during the entire activity, is used as implicit feedback for the rule based filter which makes a selection based on intensity. 


\section{Conclusions}

This study evaluated the suitability of wearables and smartphones for heart rate measurements and the automatic recognition of activities. Experiments showed that specialized devices, using a sensor with chest strap, produce very accurate heart rate measurements, similar to devices that are approved for medical purposes. Measurements with the used fitness tracker and smart watch showed very accurate results in conditions with little physical movement, e.g., in a state of rest. In contrast, a discrepancy in the measurements is witnessed during periods with a highly variable heart rate, e.g., during high intensity interval training. This low reactivity is often caused by physical movements of the device around the wrist. The device may lose the reference and a recalibration of the sensor might be required, so that no new samples are available for a few seconds. Using PPG on a smartphone resulted in fluctuating measurements, even in case of limited physical movements. Fluctuations are causes by changes in light intensity in the environment or small movements of the finger. Aggregated statistics, such as average and median, showed to be a good representation of the real heart rate. Available algorithms implementing the PPG technique are often too general because of hardware dependent characteristics. The general applicability of the algorithms allows heart rate monitoring on different smartphone devices, but reduces the accuracy. Although fitness trackers, smart watches, or PPG on smartphones are useful tools, physical movements or sudden variations in heart rate may cause inaccuracies in the heart rate measurements. For professional athletes or medical purposes, specialized devices with a chest strap might be the best choice for accurate data. Data of the accelerometer can be used to automatically recognize physical exercises such as squats or dumbbell curl by detecting peaks in the data. This allows to couple these exercises to the heart rate for further analysis, but also to assist the user in coaching tasks such as counting the number of times an exercises is performed. An eHealth recommender system can offer personal suggestions for physical activities based on personal preferences, the measured heart rate, and performed exercises. Automatic activity recognition can help the user by reducing the burden of provide input about the performed activities. These results are important for the evolution of health applications and services on the mobile platform that use data of heart rate or the accelerometer to inform, coach, or stimulate the user. In future work, the quality of the performance of the physical activity will be detected, which will allow to provide more specific feedback to the users.

Acknowledgements The authors would like to thank Enias Cailliau for his extensive research and implementation work in the context of this study.

\section{References}

1. Altini, M.: Heart Rate Variability for Training (2013). [Online] Available at: http: //www.marcoaltini.com/blog/heart-rate-variability 
2. Andoh, H., Ishikawa, T., Kobayashi, K., Kobayashi, K., Watanabe, K., Nakamura, T.: Home health monitoring system in the sleep. In: SICE 2003 Annual Conference (IEEE Cat. No.03TH8734), vol. 2, pp. 1223-1226 Vol.2 (2003)

3. Beveridge, M., Milward, D.: Definition of the high-level task specification language. deliverable d11, eu 5th framework homey project. Tech. rep., IST-2001-32434 (2003)

4. Binsted, K., Cawsey, A., Jones, R.: Generating personalised patient information using the medical record, pp. 29-41. Springer Berlin Heidelberg, Berlin, Heidelberg (1995)

5. Bodenheimer, T., Wagner, E.H., Grumbach, K.: Improving primary care for patients with chronic illness. Jama 288(14), 1775-1779 (2002)

6. Booth, F.W., Roberts, C.K., Laye, M.J.: Lack of exercise is a major cause of chronic diseases (2011)

7. Boulos, M.N.K., Brewer, A.C., Karimkhani, C., Buller, D.B., Dellavalle, R.P.: Mobile medical and health apps: state of the art, concerns, regulatory control and certification. Online journal of public health informatics 5(3), 229 (2014)

8. Campbell, M.K., DeVellis, B.M., Strecher, V.J., Ammerman, A.S., DeVellis, R.F., Sandler, R.S.: Improving dietary behavior: the effectiveness of tailored messages in primary care settings. American journal of public health 84(5), 783-787 (1994)

9. Chen, S., Lach, J., Amft, O., Altini, M., Penders, J.: Unsupervised activity clustering to estimate energy expenditure with a single body sensor. In: 2013 IEEE International Conference on Body Sensor Networks, pp. 1-6 (2013)

10. Cline, R.J., Haynes, K.M.: Consumer health information seeking on the internet: the state of the art. Health education research 16(6), 671-692 (2001)

11. Community, J.: Drools - Business rules management System (2017). [Online] Available at: http://www.drools.org/

12. Dadashi, F., et al.: A hidden markov model of the breaststroke swimming temporal phases using wearable inertial measurement units. In: 2013 IEEE International Conference on Body Sensor Networks, pp. 1-6 (2013)

13. Ekstrand, M.D., Ludwig, M., Kolb, J., Riedl, J.T.: Lenskit: a modular recommender framework. In: Proceedings of the fifth ACM conference on Recommender systems, pp. 349-350. ACM (2011)

14. El-Amrawy, F., Nounou, M.I.: Are currently available wearable devices for activity tracking and heart rate monitoring accurate, precise, and medically beneficial? Healthcare informatics research 21(4), 315-320 (2015)

15. Eysenbach, G.: Consumer health informatics. British medical journal 320(7251), 1713 (2000)

16. Fernandez-Luque, L., Karlsen, R., Vognild, L.: Challenges and opportunities of using recommender systems for personalized health education. Studies in health technology and informatics 150, 903-907 (2008)

17. Freedson, P.S., Miller, K.: Objective monitoring of physical activity using motion sensors and heart rate. Research quarterly for exercise and sport 71(sup2), 21-29 (2000)

18. Giorginoll, T., Quaglinil, C.R., Baccheschi, J.: The homey project: a telemedicine service for hypertensive patients. Personalisation for e-Health 21, 32-35 (2005)

19. Hammer, S., Kim, J., André, E.: Med-styler: Metabo diabetes-lifestyle recommender. In: Proceedings of the Fourth ACM Conference on Recommender Systems, RecSys '10, pp. 285-288. ACM, New York, NY, USA (2010)

20. Hammer, S., Seiderer, A., André, E., Rist, T., Kastrinaki, S., Hondrou, C., Raouzaiou, A., Karpouzis, K., Kollias, S.: Design of a lifestyle recommender system for the elderly: Requirement gatherings in germany and greece. In: Proceedings of the 8th ACM International Conference on PErvasive Technologies Related to Assistive Environments, PETRA '15, pp. 80:1-80:8. ACM, New York, NY, USA (2015)

21. Handel, M.J.: mhealth (mobile health)using apps for health and wellness. EXPLORE: The Journal of Science and Healing 7(4), 256-261 (2011)

22. Hayes, T.L., Abendroth, F., Adami, A., Pavel, M., Zitzelberger, T.A., Kaye, J.A.: Unobtrusive assessment of activity patterns associated with mild cognitive impairment. Alzheimer's \& Dementia 4(6), 395-405 (2008)

23. Jannach, D., Zanker, M., Felfernig, A., Friedrich, G.: Recommender Systems: An Introduction, 1st edn. Cambridge University Press, New York, NY, USA (2010)

24. Katz, L.N., Pick, A.: Clinical electrocardiography. Lea \& Febiger (1956) 
25. Khan, S.A., et al.: Gethealthyharlem.org: developing a web platform for health promotion and wellness driven by and for the harlem community. AMIA. Annual Symposium proceedings / AMIA Symposium. 2009, 317-321 (2009)

26. Kim, J.: Wearable heart rate monitors enter the consumer mainstream (2014). [Online] Available at: http://internetofthingsagenda.techtarget.com/feature/ Wearable-device-heart-rate-monitoring-entering-the-consumer-mainstream

27. Kroll, R.R., Boyd, J.G., Maslove, D.M.: Accuracy of a wrist-worn wearable device for monitoring heart rates in hospital inpatients: A prospective observational study. Journal of medical Internet research 18(9), 253 (2016)

28. Lester, J., Choudhury, T., Kern, N., Borriello, G., Hannaford, B.: A hybrid discriminative/generative approach for modeling human activities. In: Proceedings of the 19th international joint conference on Artificial intelligence, pp. 766-772. Morgan Kaufmann Publishers Inc. (2005)

29. Liu, Y., Nie, L., Han, L., Zhang, L., Rosenblum, D.S.: Action2activity: Recognizing complex activities from sensor data. In: IJCAI, pp. 1617-1623 (2015)

30. Liu, Y., Nie, L., Liu, L., Rosenblum, D.S.: From action to activity: Sensor-based activity recognition. Neurocomputing 181(Supplement C), 108 - 115 (2016). Big Data Driven Intelligent Transportation Systems

31. Liu, Y., Zhang, X., Cui, J., Wu, C., Aghajan, H., Zha, H.: Visual analysis of child-adult interactive behaviors in video sequences. In: 2010 16th International Conference on Virtual Systems and Multimedia, pp. 26-33 (2010)

32. Lu, Y., Wei, Y., Liu, L., Zhong, J., Sun, L., Liu, Y.: Towards unsupervised physical activity recognition using smartphone accelerometers. Multimedia Tools and Applications $\mathbf{7 6}(8), 10,701-10,719(2017)$

33. Megret, R., Szolgay, D., Benois-Pineau, J., Joly, P., Pinquier, J., Dartigues, J.F., Helmer, C.: Wearable video monitoring of people with age dementia : Video indexing at the service of helthcare. In: 2008 International Workshop on Content-Based Multimedia Indexing, pp. 101-108 (2008)

34. Mubashir, M., Shao, L., Seed, L.: A survey on fall detection: Principles and approaches. Neurocomputing 100, 144-152 (2013)

35. Omron: Automatic Blood Pressure Monitor - Model M6 Comfort IT (2015). [Online] Available at: www.omron-healthcare.com/en/support/manuals/download/ m6-comfort-it-hem-7322u-e-en

36. OpenWeatherMap: urrent weather and forecast (2017). [Online] Available at: http: //openweathermap.org/

37. Plasqui, G., Bonomi, A., Westerterp, K.: Daily physical activity assessment with accelerometers: new insights and validation studies. obesity reviews 14(6), 451-462 (2013)

38. Realm: Realm - Create reactive mobile apps in a fraction of time (2017). [Online] Available at: https://realm.io/

39. Reisner, A., Shaltis, P.A., McCombie, D., Asada, H.H.: Utility of the photoplethysmogram in circulatory monitoring. The Journal of the American Society of Anesthesiologists 108(5), 950-958 (2008)

40. Strecher, V., Kreuter, M., Den Boer, D., Kobrin, S., Hospers, H., Skinner, C.: The effects of computer-tailored smoking cessation messages in family practice settings. The Journal of family practice $\mathbf{3 9}(3)$, 262-270 (1994)

41. Strecher, V.J., et al.: The effects of computer-tailored smoking cessation messages in family practice settings. Journal of Family Practice 39(3), 262-270 (1994)

42. van Velsen, L., Beaujean, D.J., van Gemert-Pijnen, J.E.: Why mobile health app overload drives us crazy, and how to restore the sanity. BMC medical informatics and decision making 13(1), 23 (2013)

43. de Vries, H., Brug, J.: Computer-tailored interventions motivating people to adopt health promoting behaviours: Introduction to a new approach. Patient Education and Counseling 36(2), 99-105 (1999)

44. Weippert, M., et al.: Comparison of three mobile devices for measuring $r-r$ intervals and heart rate variability: Polar s810i, suunto t6 and an ambulatory ecg system. European journal of applied physiology 109(4), 779-786 (2010)

45. Wu, G., Xue, S.: Portable preimpact fall detector with inertial sensors. IEEE Transactions on Neural Systems and Rehabilitation Engineering 16(2), 178-183 (2008) 
46. Wu, W., Au, L., Jordan, B., Stathopoulos, T., Batalin, M., Kaiser, W., Vahdatpour, A., Sarrafzadeh, M., Fang, M., Chodosh, J.: The smartcane system: An assistive device for geriatrics. In: Proceedings of the ICST 3rd International Conference on Body Area Networks, BodyNets '08, pp. 2:1-2:4. ICST (Institute for Computer Sciences, SocialInformatics and Telecommunications Engineering), ICST, Brussels, Belgium, Belgium (2008) 